\title{
Acidentes e Violências: caracterização dos atendimentos no pronto-socorro de um hospital universitário
}

\section{Accidents and Violence: characteristics of the medical cares in the emergency room's university hospital}

José Luís Guedes dos Santos

Acadêmico de Enfermagem da Universidade Federal de Santa Maria - UFSM. Bolsista do Observatório de Acidentes e Violência do Hospital Universitário de Santa Maria - HUSM.

Endereço: R. Franklin Bittencourt Filho, 65, Camobi, CEP 97105-150, Santa Maria, RS, Brasil.

E-mail: joseennfermagemळyahoo.com.br

\section{Estela Regina Garlet}

Enfermeira. Professora Substituta da UFSM. Mestranda Programa de Pós-Graduação em Enfermagem da Escola de Enfermagem da Universidade Federal do Rio Grande do SUl - UFRGS.

Endereço: Avenida Integração/2290, Centro, CEP 98150-000, Pinhal Grande, RS, Brasil.

E-mail: estelagarletæhotmail.com

\section{Raquel Basso Figueira}

Enfermeira do pronto-socorro do Hospital Universitário de Santa Maria (HUSM). Coordenadora do Observatório de Acidentes e Violência do HUSM.

Endereço: Av. Roraima, prédio 22, Hospital Universitário de Santa Maria, Pronto-Socorro, Camobi, CEP 97105-900, Santa Maria, RS, Brasil.

E-mail: raquelbassofigueira®bol.com.br

\section{Suzinara Beatriz Soares de Lima}

Enfermeira do pronto-socorro do Hospital Universitário de Santa Maria. Doutoranda em Enfermagem pela Escola de Enfermagem Ana Nery/Universidade Federal do Rio de Janeiro.

Endereço: Av. Roraima, prédio 22, Hospital Universitário de Santa Maria, Pronto-Socorro, Camobi, CEP 97105-900, Santa Maria, RS, Brasil.

E-mail: suzibslimaœyahoo.com.br

\section{Adelina Giacomelli Prochnow}

Enfermeira. Professora Doutora do Departamento de Enfermagem da Universidade Federal de Santa Maria .

Endereço: R. Franklin Bittencourt Filho, 65, Camobi, CEP 97105-150, Santa Maria, RS, Brasil.

E-mail: agp.sma®terra.com.br

\section{Resumo}

Este artigo, de caráter exploratório-descritivo, tem como objetivo caracterizar os atendimentos por acidentes e violências realizados no pronto-socorro de um hospital universitário localizado no interior do Rio Grande do Sul (RS). Os dados foram obtidos a partir dos registros de um sistema de vigilância, denominado "Observatório de Acidentes e Violência", existente nos serviços de urgência e emergência no RS, que atuam como sentinela desses agravos. Os resultados mostraram que os adultos jovens, com baixo nível de instrução e não-trabalhadores são as principais vítimas de acidentes e violências. Quanto ao tipo de ocorrência, destacaram-se os acidentes de trânsito e acidentes domésticos, gerando como agravos mais constantes ferimentos na cabeça e fraturas de fêmur. Com relação ao sexo, embora para alguns tipos de acidentes e violências a predominância tenha variado entre eles, o conjunto dos dados mostrou maior vulnerabilidade masculina, em especial nos casos de violência interpessoal. Nesse sentido, as consequências dos acidentes e violências para o sistema de saúde e para a sociedade apontam a necessidade de aprimoramento dos sistemas de informações de morbimortalidade por causas externas, visando subsidiar políticas públicas de prevenção e melhoria no atendimento às vítimas.

Palavras-chave: Acidente; Violência; Morbidade; Prevenção; Controle; Serviços médicos de emergência. 


\section{Abstract}

This article, of an exploratory-descriptive character, aims to characterize the medical care provided in cases of accidents and violence in the emergency room of a university hospital, located in the interior of the state of Rio Grande do Sul (RS). The data were obtained from the registers of a monitoring system called “Observatório de Acidentes e Violência” (Observatory of Accidents and Violence), which exists in the urgency and emergency services in RS and acts as a sentry of these injuries. The results showed that young adults who are non-workers and have low schooling are the main victims of accidents and violence. The most frequent types of occurrence were domestic accidents and traffic accidents, generating as the most constant injuries wounds in the head and femur fractures. With regard to the sex, although for some types of accidents and violence the predominance varied between them, the set of data showed greater masculine vulnerability, especially in cases of interpersonal violence. Thus, the consequences of accidents and violence for the health system and the society point to the need of improving the information systems of morbimortality caused by external causes, in order to subsidize public prevention policies and improve the care provided for the victims.

Keywords: Accident; Violence; Morbidity; Prevention; Control; Emergency Medical Services.

\section{Introdução}

No Brasil, as mortes por acidentes e violências, que recebem da Organização Mundial da Saúde (OMS) o nome genérico de "causas externas", ocupam o segundo lugar no perfil da mortalidade geral e é a primeira causa de óbitos na faixa etária de 5 a 49 anos. Entre 1991 e 2000, cerca de 1.118 .651 pessoas morreram por esses motivos em nosso país. Desse total, 369.068 foram a óbito por homicídios; 62.480 , por suicídio e 309.212, por acidentes e violências no trânsito e nos transportes (Brasil, 2005).

A magnitude desses dados ressalta a dimensão da temática no panorama complexo dos problemas sociais do país. As lesões, os traumas e as mortes decorrentes de acidentes e violências geram altos custos emocionais e sociais. Dentre eles, estão os prejuízos econômicos em virtude dos dias de ausência no trabalho, os danos mentais e emocionais incalculáveis causados às vítimas e suas famílias, que respondem por anos de produtividade ou vida perdidos (Brasil, 2005; Minayo, 2005).

Ao sistema de saúde, as consequências da violência evidenciam-se no aumento de gastos com emergência, assistência e reabilitação, muito mais onerosos que a maioria dos procedimentos médicos convencionais. Cálculos estimam que cerca de 3,3\% do PIB brasileiro são gastos com os custos diretos da violência, cifra que sobe para 10,5\% quando se incluem custos indiretos e transferências de recursos (Briceño-León, 2002). Esse autor ainda comenta que dados do Banco Interamericano de Desenvolvimento destacam que na América Latina os danos e as transferências de recursos que se processam por causa da violência atingem 14,2\% do PIB da região e equivalem a 168 milhões de dólares.

No Rio Grande do Sul, em 2002, a mortalidade por causas externas foi a mais acentuada na faixa etária de 1 a 39 anos, sendo esses óbitos decorrentes de homicídios e acidentes de transporte, numa tendência crescente. Nos últimos anos, as causas externas são responsáveis pelos maiores índices do indicador referente aos anos potenciais de vida perdidos, visto que atingiram uma população extremamente jovem (Rio Grande do Sul, 200oa, 200ob, 2002).

1 Causas externas: designam os homicídios, suicídios, acidentes fatais e ventos mórbidos referentes a acidentes de trânsito, quedas, afogamentos, exposição à fumaça e fogo, envenenamentos e intoxicações, agressões, intervenções legais e operações de guerra, entre "outras causas" (Deslandes, 2002). 
Diante desse panorama, como medida governamental, a Portaria n. ${ }^{0} 737 / \mathrm{GM}$ de 16 de maio de 2001 institui, nacionalmente, a Política de Redução da Mortalidade por Acidentes e Violência, que se propõe a ser instrumento norteador da atuação do setor saúde nesse contexto, assim:

[...] adota como expressão desses eventos a morbimortalidade devida ao conjunto das ocorrências acidentais e violentas que matam ou geram agravos à saúde e que demandam atendimento nos serviços de saúde. Acresce a esse grupo de eventos aqueles que, mesmo não chegando aos serviços de saúde, são do conhecimento de outros setores da sociedade como políticas, hospitais não credenciados ao Sistema Único de Saúde - SUS, entre outros (Brasil, 2001, p. 1).

Neste quadro epidemiológico, inserem-se os serviços e as preocupações com a intervenção na realidade. Particularmente, a morbidade por acidentes e violências não se apresenta de forma clara. Constata-se a falta de registros e do próprio reconhecimento de agravos dessa natureza, considerados a partir dos atendimentos da Atenção Básica de Saúde e em Serviços de Emergência, o que inviabiliza o conhecimento dessa realidade nos sistemas locais e, assim, as ações de intervenção e as estratégias de controle e prevenção ficam prejudicadas (Lopes e col., 2004).

Dessa forma, este estudo tem como objetivo caracterizar os atendimentos por acidentes e violências realizados no pronto-socorro de um hospital universitário no interior do Rio Grande do Sul (RS). Com isso, espera-se melhor identificar a realidade e contribuir para a definição de novas estratégias de atuação nessa área, por meio da melhoria da estruturação dos serviços e da implementação de ações de controle e prevenção de acidentes e violências.

\section{Metodologia}

Foi realizado um estudo do tipo exploratório-descritivo com abordagem quantitativa. O estudo exploratório-descritivo permite o aprofundamento de um determinado problema e a abordagem quantitativa possibilita a caracterização e a quantificação dos objetivos propostos por meio de medidas obtidas a partir de análises estatísticas (Richardson, 1999).
O cenário da pesquisa foi o pronto-socorro (PS) de um hospital universitário do interior do estado do Rio Grande do Sul (RS), certificado como de ensino, e referência terciária para os atendimentos de alta complexidade para cerca de 46 municípios na sua área de abrangência. Essa instituição presta serviços assistenciais em várias especialidades médicas e desempenha a função de capacitação e qualificação para alunos de graduação e de pós-graduação, além de ser centro de programação e manutenção de ações voltadas à saúde das comunidades locais e regionais e desenvolver programas específicos de assistência, devidamente integrada à rede regional de saúde.

O referido serviço de pronto-socorro foi estruturado para atender urgências e emergências, mas devido ao aumento significativo da demanda por atendimento, desempenha também triagem para os demais serviços da cidade e região.

Os dados utilizados para análise foram obtidos a partir dos registros de morbimortalidade do "Observatório de Acidentes e Violência”. Trata-se de uma iniciativa da Secretaria do Estado do Rio Grande do Sul, que instituiu em 2001, em hospitais gaúchos, tidos de referência no atendimento a acidentes e violências, pontos de notificação e registro desses agravos. 0 projeto faz parte do Sistema de Vigilância Epidemiológica em Saúde do Trabalhador, que integra o Sistema de Vigilância Epidemiológica em Saúde do Trabalhador da Secretaria Estadual de Saúde (SVST/SES), do Sistema Comunicação de Acidentes do Trabalho (CAT) e do Instituto Nacional do Seguro Social (INSS). As informações foram obtidas a partir dos instrumentos de notificação que alimentam um banco de dados gerenciado pelo SVST.

Esse sistema de vigilância é alimentado por meio da busca diária dos atendimentos por acidentes e violências realizados na referida instituição. Para isso, há um formulário específico, o Relatório Individual de Notificação de Agravos Decorrentes de Violência (RINAV), que é composto de variáveis de identificação do indivíduo e do agravo do qual ele foi vítima. O preenchimento desse formulário é realizado mediante a consulta dos prontuários dos vitimados e/ou entrevista direta com eles. Isso nem sempre é possível devido a situações, como, por exemplo, o déficit de consciência da vítima, a ausência de acompanhante e a curta permanência da vítima no serviço, seja por transferência 
para outro setor da instituição seja por alta hospitalar após estabilização do quadro traumático.

O período de análise abrangeu os meses de janeiro de 2004 a dezembro de 2006. Nesse intervalo, foram encontrados 468 registros de acidentes e violências no banco de dados no Observatório de Acidentes e Violência. Salienta-se que esse número não representa a totalidade dos atendimentos, visto que ainda não se tem instituído uma sistemática para notificação desses agravos e um quantitativo de pessoal suficiente para desenvolver essa atividade.

Para este trabalho, estruturaram-se como categorias de estudo: faixa etária, sexo, escolaridade, situação de trabalho, procedência das vítimas, local e tipo de ocorrência, vínculo empregatício e agravo ocasionado. No intuito de facilitar a apresentação e a compreensão desses resultados foram elaboradas e utilizadas tabelas ilustrativas.

\section{Resultados}

Conforme mostra a Tabela 1, entre 2004 e 2006, a faixa etária que predominou entre as vítimas de acidentes e violências, com 129 registros, foi a de 20 aos 30 anos, sendo 99 homens e 30 mulheres; já entre os 30 e 40 anos foram atendidos 56 homens e 30 mulheres, totalizando 86 casos. A análise total segundo o sexo apontou que $(74,78 \%)$ dos atendimentos eram referentes a indivíduos do sexo masculino.

No que tange a escolaridade dos atendidos, a maioria (30,55\%) possuía Ensino Fundamental incompleto; 6,o8\%, o Ensino Médio incompleto; 4,37\%, Ensino Fundamental completo; 4,18\%, Ensino Médio completo; 1,71\%, superior incompleto; e, o,38\%, superior completo.

Com relação à situação de trabalho dos vitimados, $34,91 \%$ eram não trabalhadores, por serem jovens estudantes ou estarem desempregados; $27,32 \%$ eram trabalhadores informais sem carteira assinada; $15,75 \%$ eram profissionais autônomos; e, 8,92\%, aposentados.

Quanto à procedência das vítimas atendidas, a maior parte delas (51,70\%) era proveniente do município sede, sendo as demais originárias de municípios vizinhos, que têm esse serviço de pronto-socorro como referência para urgências e emergências, totalizando 48,30\%.

A caracterização quanto ao local de ocorrência dos agravos revelou, como pode ser observado na Tabela 2, que a maior parte deles ocorreu em espaços públicos
Tabela I - Características socioeconômicas das vítimas de acidentes e violências

\begin{tabular}{l|c|c|c|c} 
Caracteristicas socioeconômicas & \multicolumn{3}{|c|}{ N } & \multicolumn{3}{c}{$\%$} \\
Faixa etária* & M & F & M & F \\
\hline 20-30 anos & 99 & 30 & 21,15 & 6,41 \\
\hline $30-40$ anos & 56 & 30 & 11,96 & 6,41 \\
\hline Sexo & \multicolumn{3}{|c|}{350} & 74,78 \\
\hline Masculino & \multicolumn{3}{|c}{118} & 25,22 \\
\hline Feminino & \multicolumn{3}{|c}{} \\
\hline
\end{tabular}

\section{Escolaridade*}

\begin{tabular}{l|c|c}
\hline Fundamental incompleto & 142 & 30,55 \\
\hline Médio incompleto & 28 & 6,08 \\
\hline Fundamental completo & 20 & 4,37 \\
\hline Médio completo & 19 & 4,18 \\
\hline Superior incompleto & 8 & 1,71 \\
\hline Superior completo & 2 & 0,38 \\
\hline Situação de trabalho & \multicolumn{1}{|c|}{163} & 34,91 \\
\hline Não-trabalhadores & 127 & 27,32 \\
\hline Profissionais autônomos & 73 & 15,75 \\
\hline $\begin{array}{l}\text { Trabalhadores informais } \\
\text { (sem carteira assinada) }\end{array}$ & 55 & 11,75 \\
\hline Aposentados & & \\
\hline
\end{tabular}

* Considerando a multiplicidade de possibilidades nessa categoria, optou-se por não se expor na tabela a totalidade dos dados. Assim, foram listados apenas aquelas com maior valor quantitativo.

(39,10\%) ou no domicílio (32,47\%), sendo que apenas $5,34 \%$ dos casos ocorreram no trabalho.

No que se refere ao tipo de ocorrência, as mais registradas foram: acidentes de trânsito $(16,51)$, acidente doméstico (11,57\%), violência física (11,01\%) e acidentes de trabalho $(7,21 \%)$. Entre as consequências decorrentes desses episódios, destacam-se os agravos: ferimentos da cabeça (9,69\%), fraturas de fêmur (7,22\%), traumatismos na região superficial da cabeça $(5,32 \%)$, fraturas da perna, incluindo tornozelo $(4,75 \%)$, fraturas no nível do punho e da mão $(3,99)$, fraturas do punho e da mão $(3,61 \%)$ e fraturas do antebraço (3,04\%).

A análise da relação do sexo e o tipo de ocorrência revelou que do total de vítimas do sexo masculino (74,78\%), 13,28\% foram atendidas em decorrências acidentes de trânsito, $8,54 \%$ por violência física e 7,02\% em virtude de acidentes de trabalho. As mulhe- 
Tabela 2 - Caracterização da ocorrência, conforme local, tipo e agravos causados

Caracteristicas da ocorrência

\begin{tabular}{l|l}
$\mathrm{N}$ & $\%$
\end{tabular}

Local*

\begin{tabular}{l|c|c}
\hline Espaços públicos & 182 & 39,10 \\
\hline Domicílio & 151 & 32,47 \\
\hline Trabalho & 25 & 5,34 \\
\hline
\end{tabular}

Tipo*

\begin{tabular}{l|c|c}
\hline Acidente de trânsito & 77 & 16,51 \\
\hline Acidente doméstico & 54 & 11,57 \\
\hline Violência física & 51 & 11,01 \\
\hline Acidente de trabalho & 34 & 7,21 \\
\hline
\end{tabular}

Agravos causados*

\begin{tabular}{l|c|c}
\hline Ferimento na cabeça & 45 & 9,69 \\
\hline Fratura de fêmur & 34 & 7,22 \\
\hline $\begin{array}{l}\text { Traumatismo na região } \\
\text { superficial da cabeça }\end{array}$ & 25 & 5,32 \\
\hline Fratura da perna, incluindo tornozelo & 22 & 4,75 \\
\hline Fratura do nível do punho e da mão & 19 & 3,99 \\
\hline Fratura do punho e da mão & 17 & 3,61 \\
\hline Fratura do antebraço & 14 & 3,04 \\
\hline
\end{tabular}

res $(25,22 \%)$ foram atendidas, na maioria das vezes, em virtude de acidentes domésticos (6,83\%), acidentes de trânsito $(3,23 \%)$ e violência física $(2,47 \%)$. Ressalta-se que os dados referentes à violência sexual contra mulheres não foram computados nesta análise, uma vez que o esse tipo de atendimento ocorre diretamente no Centro Obstétrico da instituição na qual o estudo foi realizado.

\section{Discussão}

Os índices encontrados revelam coerência com outros estudos realizados em relação aos acidentes e os casos de violência atendidos nos diversos serviços públicos de saúde em nosso país.

A análise dos dados apontou que o maior número de atendimentos por acidentes e violências está concentrado entre a parcela da população na faixa etária de 20 a 30 anos. Esse resultado vai ao encontro do estudo de Máximo (2006), que aponta os jovens como a parcela da população mais sujeita a morte por fato- res externos: $60,4 \%$ dos óbitos na faixa etária entre 16 e 24 anos são decorrentes de homicídios, acidentes de trânsito e suicídios.

Com relação ao sexo, observa-se uma predominância masculina, o que demonstra coerência com a literatura, que aponta altos índices de morbimortalidade masculina por acidentes e violências. As representações históricas e constitutivas das culturas de gênero explicam, em algumas situações, a maior vulnerabilidade dos homens em relação a esses agravos. Para os casos de violência, por exemplo, a delinquência, o envolvimento com drogas e a demonstração de poder ou superioridade física justificam a maioria masculina nesses tipos de eventos (Sant'Anna e col., 2005).

Quanto à escolaridade das vítimas, os dados apontam que a maioria delas não possuía o Ensino Fundamental completo. $O$ baixo grau de escolaridade reflete a condição socioeconômica da região estudada, as dificuldades de acesso a educação, trabalho, saúde, lazer e cultura restringem as possibilidades de melhoria das condições de vida e saúde. As desigualdades sociais tornam turbulentas as relações entre as diversas camadas socioeconômicas e avalizam alguns aspectos das vulnerabilidades. As especificidades de cada grupo social aliado à incapacidade de suprir essas necessidades denotam a concentração da pobreza e o aumento das transgressões sociais (Abramovay e Pinheiro, 2003).

Esse fato repercute diretamente na condição de trabalho dos vitimados, pois, com relação a esse item, a grande maioria deles referiu não possuir vínculo empregatício, ora por não compor o mercado de trabalho ainda, ora por encontrar-se em situação de desemprego. Entre os trabalhadores, destacaram-se os que desenvolviam suas atividades laborais na informalidade. Assim, pode-se inferir que há uma estreita relação entre acidentes e violências e a situação socioeconômica que envolve a sua ocorrência.

A distribuição dos casos pelo local de ocorrência mostra que os locais públicos foram onde ocorreu o maior número de acidentes e casos de violência, seguidos pelo domicílio e o ambiente de trabalho. 0 local público ocupou o primeiro lugar em registros de ocorrência, em virtude dos acidentes de trânsito. Em segundo lugar, apareceram os acidentes domésticos, representados principalmente pelas quedas decorrentes da perda de equilíbrio e/ou escorregões. 
Pelo exposto, vale ressaltar os dados do Sistema de Internações Hospitalares/SUS, que apontam os acidentes de transporte, com 29.640 (25,0\%), como uma das principais causas de mortalidade, somando 74.983 $(63,3 \%)$ das mortes classificadas na categoria causas externas em 200o, sendo 652.249 (5,2\%) o número de internações hospitalares que decorrem do mesmo motivo no território nacional (Gawryszewski e col., 2004).

As consequências das ocorrências atendidas foram as mais variadas, destacando-se os ferimentos, traumatismos e fraturas na região da cabeça, fêmur, punho e mão. As altas taxas de acidentes ocorridos em via pública caracterizam a predominância desses tipos de lesões. 0 estudo de Gawryszewski e colaboradores (2004) apontou esses agravos como os mais importantes em relação aos acidentes de trânsito e transporte. As escoriações e os ferimentos leves relacionam-se a acidentes de menor intensidade.

A análise comparativa entre sexo e tipo de ocorrência revelou maior número de homens vítimas de acidentes de trânsito, o que reflete uma maior vulnerabilidade masculina em relação aos agravos por acidentes e violência em via pública. As mulheres em sua maioria são vítimas de acidentes domésticos, o que tem relação com as atividades desenvolvidas por elas, na família e na vida social, que muitas vezes colaboram para a ocorrência de eventos, particularmente os acidentais. A violência doméstica mantém-se, na maioria das vezes, velada ou subnotificada. 0 medo das vítimas de denunciar os agressores e o descaso das autoridades estimulam a subnotificação e prejudicam a qualidade da informação.

Esses números vão ao encontro do estudo de Minayo e Souza (1999), em que são reafirmadas a importante carga social desses agravos pelas vidas perdidas, sequelas e por onerarem a sociedade com custos diretos e indiretos. A discussão sobre os danos à saúde provocados pelos acidentes, em especial os de trânsito e de transporte, tem estimulado pesquisadores a explicar os motivos para sua ocorrência e estabelecer medidas preventivas.

Segundo Drumond e Abrantes (2001), além de levar à morte mais de 100.ooo brasileiros a cada ano, estimase que, para cada morte devido a traumatismos, três indivíduos permanecem definitivamente com sequelas.

Diante do panorama apresentado, corrobora-se com Whitaker e colaboradores (1998) quando eles afirmam que os custos sociais decorrentes do trauma aliados à elevação dos índices de mortalidade por acidentes e violência têm apontado a necessidade de ações de prevenção e assistência em todos os níveis de atendimento para minimizar essa problemática.

Para tanto, faz-se necessário, como aponta a Política Nacional de Redução da Morbimortalidade por Acidentes e Violências, que a prestação do atendimento às vitimas desses agravos disponha de uma rede organizada de serviços do SUS, de forma que sejam diagnosticadas essas ocorrências entre os usuários e acolhidas demandas, prestando-lhes atenção de qualidade e resolutiva, desde o primeiro nível de atenção.

Essa rede de atendimento pressupõe uma interlocução entre o setor saúde e outras áreas, como a jurídica e a de segurança, da capacitação dos profissionais para identificar as situações, especialmente de violências, fazer o acompanhamento e acionar os serviços existentes que são necessários em cada caso, além da assistência em todos os níveis por meio da definição de mecanismos de referência entre os serviços (Brasil, 2001).

Dessa forma, no intuito de alcançar essa rede entre os serviços, destaca-se a necessidade do desenvolvimento de estratégias integradoras, com foco em cada realidade epidemiológica, tanto no sentido da efetivação das políticas existentes com relação à prevenção de acidentes e violências quanto na capacitação dos profissionais para o atendimento das vítimas desses agravos nos serviços de urgência e emergência.

\section{Considerações Finais}

A violência, por muito tempo, foi abordada pela área da saúde como um problema pertinente à segurança pública ou à ordem social, permanecendo como algo externo a sua agenda, sendo classificada até os dias de hoje, pela Classificação Internacional de Doenças (CID), com a denominação de "Causas Externas": que contempla todos os agravos à saúde que causam morbidade ou mortalidade de forma acidental ou intencional (Deslandes, 2002).

No entanto, as causas externas a partir do final dos anos 1980 figuram como uma das principais causas de morte da população brasileira, chegando, em 1989, a 15,35\% das mortes ocorridas no país, tornandose, desde então, a segunda causa de óbito na popula- 
ção e a primeira entre pessoas nas faixas de 5 a 49 anos (Deslandes, 2002).

Nesse sentido, as consequências dos acidentes e violências para o sistema de saúde e para a sociedade apontam a necessidade de aperfeiçoamento do sistema de informações de mortalidade e morbidade por causas externas, visando subsidiar políticas públicas para a prevenção do problema e o atendimento das vítimas.

A partir desse pronto socorro de um hospital público e universitário, por meio da descrição das principais características da morbimortalidade por esses agravos entre as vítimas que procuraram atendimento no serviço entre janeiro de 2004 e dezembro de 2006.

Os achados indicam que a população jovem é mais vulnerável aos agravos por acidentes e violência no serviço de atendimento analisado. A vulnerabilidade física, imaturidade e curiosidade, características dessa fase, contribuem, mas não justificam os altos índices nessas faixas etárias. Medidas educativas e preventivas, certamente poderiam ajudar a reduzir essas taxas.

Embora para alguns tipos de acidentes e violências a predominância tenha variado entre os sexos, o conjunto dos dados mostrou maior vulnerabilidade masculina. Em especial nos casos de violência interpessoal, o sexo masculino é ainda mais vulnerável.

Quanto ao local de ocorrência, os espaços públicos apareceram em primeiro lugar, seguidos do ambiente domiciliar. Assim, segundo Gawryszewski e colaboradores (2004), estabelecer o “local de ocorrência” é fundamental para a construção de estratégias de prevenção.

Para consolidar uma perspectiva de atendimento de emergência seria necessário investir em alguns aspectos, dentre os quais se pode destacar a melhoria do registro hospitalar dos casos a fim de subsidiar o planejamento de ações futuras. 0 registro detalhado permitiria identificar os tipos de contextos sociais em que mais ocorrem os acidentes e violências e serviria como "mapa" para indicar ou confirmar onde os diferentes setores públicos deveriam agir (Deslandes, 2002).

Nesse sentido, este estudo dá um importante passo na descrição desses eventos e propõe, para investigações posteriores, uma análise mais apurada de suas diversas tipologias. Ressalta-se, ainda, a necessidade da criação e do desenvolvimento de formulários de registro que impeçam o ocultamento de dados, a fim de viabilizar ações preventivas, que, caso venham a ser implantadas, possam ser realmente efetivas para que a violência, em qualquer forma de expressão, seja desnudada e combatida por meio do planejamento e de decisões institucionais eficazes.

\section{Referências}

ABRAMOVAY, M.; PINHEIRO, L. C. Violência e vulnerabilidade social. In: FRAERMAN, A. (Ed.). Inclusión social y desarrollo: presente y futuro de la Comunidad IberoAmericana. Madrid: Comunica, 2003.

BRASIL, Ministério da Saúde. Política Nacional de Redução da Morbimortalidade por Acidentes e Violências. Portaria MS/GM n ${ }^{0} 737$ de 16/05/o1 publicada no DOU n ${ }^{0} 96$ seção IE, de 18/05/o1. Série $\varepsilon$. Legislação de Saúde; n. 8. Brasília: Ministério da Saúde, 2001.

BRASIL, Ministério da Saúde. Análise dos dados de mortalidade no Brasil e regiões, 2002. Boletim do Sistema de Informações sobre Mortalidade. Brasília: Ministério da saúde, DF, 2002. Disponível em: <http:/ /www.saúde.gov.br/vigilanciaepidemiológica>.

Acesso em: 05 maio 2006.

BRASIL. Ministério da Saúde. Secretaria de Vigilância em Saúde. Impacto da violência na saúde dos brasileiros. Brasília, DF, 2005.

BRICEÑO-LEÓN, R. (Org.). Violencia, sociedady justicia en América Latina. Buenos Aires: Clacso, 2002.

DESLANDES, S. F. Frágeis deuses: profissionais da emergência entre os danos da violência e a recriação da vida. Rio de Janeiro: Fiocruz, 2002.

DRUMOND, D. A. F.; ABRANTES, W. L. Tipos de trauma: o politraumatizado. In: FREIRE, E. (Ed.). Trauma: a doença dos séculos. São Paulo: Atheneu, 2001. v. 1, p. 451-549.

GAWRYSZEWSKI, V. P.; KOIZUMI, M. S.; MELLO

JORGE, M. H. P. Mortes e internações por causas externas entre os idosos no Brasil: o desafio de integrar a saúde coletiva e atenção individual. Revista da Associação Médica Brasileira, São Paulo, v. 5o, n. 1, p. 97-103, 2004. 
LOPES, M. J. M. et al. Observatório de causas externas na rede básica de saúde do município de Porto Alegre. Porto Alegre: UFRGS, EENF, 2004.

MÁXIMO, W. Mortalidade de jovens leva Brasil ao terceiro lugar em homicídios entre 84 países. Brasília, DF: Agência Brasil, 20o6. Disponível em: <http://www.agenciabrasil.gov.br/noticias/2006/11/ 16/materia.2006-11-16.2148679163/view>. Acesso em: 10 jan. 2007.

MINAYO, M. C. S. Violência: um problema para a saúde dos brasileiros. In: BRASIL. Ministério da Saúde. Secretaria de Vigilância em Saúde. Impacto da violência na saúde dos brasileiros. Brasília, DF, 2005. p. 9-42.

MINAYO, M. C. S.; SOUZA, E. R. É possível prevenir a violência?: reflexões a partir do campo da saúde pública. Ciência e Saúde Coletiva, Rio de Janeiro, v. 4, n. 1, p. 7-32, 1999.

RICHARDSON, R. J. Pesquisa social: métodos e técnicas. 3. ed. São Paulo: Atlas, 1999.
RIO GRANDE DO SUL. Secretaria da Saúde. Estatísticas de saúde: mortalidade 1998 e 1999. Porto Alegre: Coordenadoria de Informações em Saúde, 200oa. v. 24.

RIO GRANDE DO SUL. Secretaria da Saúde. Estatísticas de saúde: mortalidade 200o. Porto Alegre: Coordenadoria de Informações em Saúde, 20oob. v. 25.

RIO GRANDE DO SUL. Secretaria da Saúde. Estatísticas de saúde: mortalidade 2001. Porto Alegre: Coordenadoria de Informação em Saúde, 2002. v. 26.

SANT’ANNA, A.; AERTS, D.; LOPES, M. J. M. Homicídios entre adolescentes no sul do Brasil: situações de vulnerabilidade segundo seus familiares. Cadernos de Saúde Pública, Rio de Janeiro, v. 21, n. 1, p.120-129, 2005.

WHITAKER, I. Y.; GUTIÉRREZ, M. G. R.; KOIZUMI, M.

S. Gravidade do trauma avaliada na fase préhospitalar. Revista da Associação Médica Brasileira, São Paulo, v. 44, p. 111-119, abr./jun. 1998. 